\title{
Antimutagenic potentials of $L$-tyrosine and its metabolites on the genotoxic activity induced by methyl methanesulfonate
}

\author{
Merell Pabilona Billacura ${ }^{1, *}$, Lydia Micabalo Bajo ${ }^{2}$ \\ ${ }^{1}$ Mindanao State University-Main Campus, Marawi, Lanao del Sur 9700, Philippines \\ ${ }^{2}$ Mindanao State University-Iligan Institute of Technology, Iligan 9200, Philippines
}

\section{A R T I C L E IN F O}

\section{Article history:}

Received 6 February 2017

Received in revised form

9 September 2017

Accepted 14 September 2017

Keywords:

Peripheral blood micronucleus assay

Bone marrow micronucleus assay

Ames test

Chromosome aberration

\begin{abstract}
A B S T R A C T
$L$-Tyrosine an aromatic amino acid can be a potential chemical agent in treating Parkinson's disease. In this study, the potential of $L$-Tyrosine and its metabolites to inhibit the genotoxicity of methyl methanesulfonate (MMS) was assessed using in vivo and in vitro assays. In vivo test peripheral blood and bone marrow micronucleus assays, the antimutagenic potential of $L$ Tyrosine and its metabolites, $L$-DOPA, dopamine and epinephrine, to combat the breaking chromosome potential of MMS were evaluated using albino mice, in which the test compounds were administered orally through gavage with a double dosage regimen while in vitro assay Ames test, Salmonella typhimurium standard tester strains TA98 and TA104 were utilized as its test microorganisms. Based on the results using One-way ANOVA with square root transformation of raw data and Duncan's Multiple Range Test, it shows that the difference in the treatment means between $L$-Tyrosine and its metabolites are much lower and significantly different $(P$ value $<0.05)$ from MMS which suggests that treatment samples lack the capacity of forming micronucleated polychromatic erythrocytes (MPCEs). It is also evident that the occurrences of MPCEs were observed clearly in MMS and was the highest. Generally, results suggest that $L$-Tyrosine and its metabolites lower the genotoxic activity of MMS and hence, it can be considered to have an antimutagenic potential against chemically induced mutation. The number of revertants induced by MMS were significantly reduced, and epinephrine has the lowest activity in reducing and inhibiting the number of revertants induced by MMS while both $L$-Tyrosine and $L$-DOPA showed the highest activity.
\end{abstract}

(C) 2017 The Authors. Published by IASE. This is an open access article under the CC BY-NC-ND license (http://creativecommons.org/licenses/by-nc-nd/4.0/).

\section{Introduction}

In genetic toxicology, studying of the extent of DNA damage at the chromosome level is very important because it is an important phase in carcinogenesis. Micronucleus assay is an important tool in assessing the chromosome damage inasmuch as it reliably measure both the chromosome loss and chromosome damage (Fenech, 2000).

Alkylating agents can cause cell death, mutation and cancer, are substances that alter the structure of DNA and classified as genotoxic to both somatic cells and germ cells. The biological properties and mechanism of action of alkylating agents have been extensively explored in cells and animal model

\footnotetext{
* Corresponding Author

Email Address: merelljohn@yahoo.com (M. P. Billacura) https://doi.org/10.21833/ijaas.2017.011.016

2313-626X/C 2017 The Authors. Published by IASE.

This is an open access article under the CC BY-NC-ND license

(http://creativecommons.org/licenses/by-nc-nd/4.0/)
}

systems are categorized as mutagenic, toxic, clastogenic and teratogenic (Margison et al., 2002; Magee et al. 1976). Therefore, genotoxic and mutagenic substances in the environment must be evaluated and monitored as they often induce genetic toxicities and abnormalities.

Antimutagens are substances which can trap genotoxins and mutagens through molecular interaction which eventually inhibit the metabolism of promutagens (Sylianco, 1998). Amino acids are essential for the protein synthesis which is required for enzymatic catalysis, control growth and differentiation, transport, storage, protection, hormones, and regulation. Studies done by Bajo (1994) shows that $L$-Tyrosine and its metabolic product dopamine did not possess direct DNA damaging capacity on $\mathrm{H}_{17} \mathrm{Rec}^{+}$and $\mathrm{M}_{45} \mathrm{Rec}^{-}$strains of Bacillus subtilis. However, they were able to reduce the genotoxicity of quinoline. It has been shown from the studies of Sylianco (Sylianco and Guevara, 1985; Sylianco and Guevara, 1989; Sylianco, 1991) that 
amino acids, such as glutamic acid, aspartic acid, lysine, histidine, arginine, and cysteine; mineral ions such as calcium, magnesium, zinc, copper, iron, and manganese inhibit and reduce the activity of some mutagens and carcinogens such as dimethylhydrazine, benzo (a) pyrene, dimethylnitrosamine, azaserine and methyl methanesulfonate. In this study, the mutagenic and antimutagenic potential of $L$-tyrosine and its metabolites were evaluated using peripheral blood and bone micronucleus assays, and Ames test.

\section{Materials and methods}

Methyl methanesulfonate (MMS) (CAS No. 66-273), 3-4-Dihydoxy-L-phenylalanine (L-DOPA) (CAS No. 59-92-7), 3-Hydroxytyramine hydrochloride (dopamine hydrochloride) (CAS No. 62-31-7), Giemsa (CAS 51811-82-6) and fetal bovine serum are analytical grade reagents and were purchased from Sigma-Aldrich Co., Singapore. The epinephrine used in the experiment was bought from a local hospital in Iligan City, Philippines.

\subsection{Dosage}

In the micronucleus test, the experimental dosage of the genotoxic substance performed in the antimutagenecity study of Bajo (1994) were used for $L$-Tyrosine (30) and dopamine (300); for epinephrine (0.1), and $L$-DOPA (150) mg per kilogram body weight. For Ames test, $L$-Tyrosine (106.60 ppm), L-DOPA (509.43 ppm), dopamine (1,132.08 ppm), epinephrine (18.87 ppm) and MMS (37.74 ppm). There were two sets of control: Methyl methanesulfonate $(10 \mathrm{mg} / \mathrm{kg})$ as the positive control and distilled water as the negative control.

\subsection{Test animals/microorganisms}

Albino mice were purchased from a local petshop in Iligan City. They were fed with commercial pellets and distilled water throughout the acclimatization and experimental periods and were subjected to a regular light/dark cycle. The drinking bottles and cages were cleaned regularly. The animals were placed in a room at ambient temperature and caged in small groups according to feeding conditions. The test microorganisms Salmonella tester strains TA98 and TA104 were obtained from the Philippine National Collection of Microorganisms (PNCM), University of the Philippines-Los Baños, Laguna, Philippines with a permission from Dr. Bruce N. Ames.

\subsection{The micronucleus test}

Mice, both female and male, aged 8-12 weeks were randomly chosen. Each experimental group contained 5 mice. Prior to drug administration, mice were fasted from food and water for 16 hours and two hours after administration, food and water ad libitum were given to the experimental mice. The micronucleus assay was performed using peripheral blood and bone marrow simultaneously from the same animal with a double dosing regimen. $L$ Tyrosine, $L$-DOPA, dopamine and epinephrine; $L$ Tyrosine, $L$-DOPA, dopamine and epinephrine plus methyl methanesulfonate respectively were administered orally twice, each with $0.20 \mathrm{~mL} / 20$ gram body weight using a $500 u \mathrm{~L}$ microsyringe as an improvised gavage, $24 \mathrm{~h}$ apart, to five mice per dosage group. Both peripheral blood and bone marrow were collected $24 \mathrm{~h}$ after the second treatment.

\subsection{Peripheral blood micronucleus assay}

The method outlined in Aida et al. (1995), CSGMT (1990), Hayashi et al. (1983) and Heddle (1973) was followed with minor modifications. Prior to the collection of the blood, the mice were collapsed using chloroform and killed through cervical dislocation. Blood was collected by cutting the tail of the mouse and a drop of blood was placed on the pre-cleaned and coded glass slides, and smeared using a cover slip then air dried. The prepared blood films were fixed in methanol then air dried again. The smeared preparations were stained with acridine orange (AO).

\subsection{Bone marrow micronucleus assay}

The method outlined in Schmid (1976) was followed with modifications. The mice were sacrificed by cervical dislocation. The femora were excised and cleaned of muscle tissues present then the bone marrow was flushed with $0.20 \mathrm{~mL}$ fetal calf serum in a micro test tube. The suspensions were centrifuged at $1000 \mathrm{rpm}$ for 5 minutes to sediment the cells; supernatants was discarded and each of the sediments were homogeneously mixed using pasteur pipette then smeared on the pre-cleaned and coded glass slides, and smeared using a cover slip. The prepared bone marrow films were air-dried and fixed in methanol then air dried overnight and was stained the following morning. Staining of slides were done in a undiluted May-Gruenwald stain for 5 minutes, then were transferred to a 50\% MayGruenwald stain for 2 minutes, after which, the slides were immediately transferred to a $15 \%$ aqueous Giemsa stain solution for 10 minutes then the back was wiped with a tissue paper.

Screening of the slides for micronucleated polychromatic erythrocytes in the peripheral blood and bone marrow cells followed using 1000x magnification. Five slides per mouse were prepared and scoring was done from 1000 cells per slide or 5000 per mouse.

\subsection{Ames test}

The method outlined in the studies conducted by Mortelmans and Zeiger (2000), Ames et al. (1973), 
and Maron and Ames (1983) was followed minor modification. To the sterile $20 \mathrm{~mL}$ test tube the following were added as follows: $0.5 \mathrm{~mL}$ of phosphate buffer, $0.1 \mathrm{~mL}$ of bacterial culture, and $0.05 \mathrm{~mL}$ or less of test solution. The mixture was mildly mixed using vortex. The mixture was incubated for 20 minutes at $37^{\circ} \mathrm{C}$. After the incubation, $2 \mathrm{~mL}$ of top agar (with $0.5 \mathrm{mM}$ histidine/biotin solution) maintained at $43-48^{\circ} \mathrm{C}$ was added. The contents of test tubes are then mixed using vortex at moderate speed for 3 seconds and poured onto the surface of minimal-glucose agar plates then tilted and rotated for even distribution of the mixture. The top agar was set aside to harden for an hour. When the top agar has hardened, the plates are inverted and placed in a $37^{\circ} \mathrm{C}$ incubator. After 48-72 hours, the plates were removed from the incubator and number of visible colonies per plate was counted. Three trials per test sample were done with three plates per trial. Controls were done side by side with the test samples with three plates per trial. Methyl methanesulfonate and sterilized distilled water was used as the positive control and negative control, respectively.

\subsection{Statistical evaluation}

Statistical analyses of the results were undertaken using the One-way Analysis of Variance
(ANOVA) with square root transformation of raw data and Duncan's Multiple Range Test (DMRT).

\section{Results and discussion}

\subsection{In vivo effects}

Micronucleus assays using peripheral blood reticulocytes and bone marrow cells were used to evaluate the antimutagenic effects of the test compounds on the known alkylating and carcinogen, methyl methanesulfonate.

Results on Table 1 show that $L$-Tyrosine, $L$-DOPA, dopamine, and epinephrine lack the capacity of forming micronucleated polychromatic erythrocytes (MPCEs) which suggests a lack of chromosome breaking effects on the peripheral blood reticulocytes and bone marrow cells. These results indicate that the test samples do not alter the structure of DNA. The number of micronucleated erythrocytes found on these substances is mainly due to the spontaneous fragmentation of the chromatin material at the level of the negative control. The positive control, methyl methanesulfonate (MMS), showed a very significant number of micronucleated polychromatic erythrocytes in comparison with the negative control, and $L$-Tyrosine and its metabolites.

Table 1: The chromosome breaking potential of $L$-Tyrosine, $L$-DOPA, dopamine, and epinephrine

\begin{tabular}{ccccc}
\hline \multirow{2}{*}{ Test Compounds } & \multicolumn{3}{c}{ Number of Micronucleated Polychromatic Erythrocytes } \\
\cline { 2 - 5 } & \multicolumn{3}{c}{ Peripheral Blood } & \multicolumn{2}{c}{ Bone Marrow } \\
& Mean Raw Data & Transformed Data & Mean Raw Data & Transformed Data \\
\hline Distilled water & 0.200 & $3.814^{\mathrm{b}} \pm 0.401$ & 0.0667 & $3.639^{\mathrm{b}} \pm 0.231$ \\
MMS & 51.067 & $27.780^{\mathrm{a}} \pm 0.153$ & 24.733 & $19.471^{\mathrm{a}} \pm 0.676$ \\
L-Tyrosine & 0.133 & $3.743^{\mathrm{b}} \pm 0.284$ & 0.133 & $3.743^{\mathrm{c}} \pm 0.284$ \\
$L$-DOPA & 0.000 & $3.536^{\mathrm{b}} \pm 0.000$ & 0.133 & $3.743^{\mathrm{c}} \pm 0.284$ \\
Dopamine & 0.0667 & $3.639^{\mathrm{b}} \pm 0.231$ & 0.933 & $4.810^{\mathrm{b}} \pm 0.635$ \\
Epinephrine & 0.0667 & $3.639^{\mathrm{b}} \pm 0.231$ & 0.133 & $3.743^{\mathrm{c}} \pm 0.284$ \\
\hline \multicolumn{2}{c}{ Note: Values are based on the average of readings of 5 slides per mouse per 5 trials } \\
\multicolumn{2}{r}{ Means having the same letter are not significantly different at $\alpha=0.05$ DMRT }
\end{tabular}

Results on Table 2 show that $L$-Tyrosine has the highest activity and epinephrine has the lowest activity in reducing the number of number of MPCEs in the peripheral blood micronucleus assay and in the bone marrow micronucleus assay, epinephrine and $L$-DOPA has the lowest activity in reducing the number of MPCE's and the activity of $L$-Tyrosine, $L$ DOPA, and dopamine reducing the number of MPCE's were insignificantly different. Generally, these results mean that L-Tyrosine and its metabolites can inhibit the chromosome breaking potential of MMS.

Table 2: Effects of $L$-Tyrosine and its metabolites on the chromosome breaking effects of methyl methanesulfonate $\left(\mathrm{CH}_{3}{ }^{+}\right)$

\begin{tabular}{ccccc}
\hline \multirow{2}{*}{ Test Compounds } & \multicolumn{3}{c}{ Number of Micronucleated Polychromatic Erythrocytes } \\
& \multicolumn{2}{c}{ Peripheral Blood } & \multicolumn{2}{c}{ Bone Marrow } \\
\cline { 2 - 5 } & Mean Raw Data & Transformed Data & Mean Raw Data & Transformed Data \\
\hline Distilled water & 0.200 & $3.814^{\mathrm{f}} \pm 0.401$ & 0.0667 & $3.639^{\mathrm{d}} \pm 0.231$ \\
MMS & 51.067 & $27.780^{\mathrm{a}} \pm 0.153$ & 24.733 & $19.471^{\mathrm{a}} \pm 0.676$ \\
$L$-Tyrosine + MMS & 1.600 & $5.690^{\mathrm{e}} \pm 1.055$ & 1.600 & $5.690^{\mathrm{c}} \pm 1.055$ \\
$L$-DOPA & 2.533 & $6.702^{\mathrm{d}} \pm 0.831$ & 2.067 & $6.187^{\mathrm{bc}} \pm 0.467$ \\
Dopamine + MMS & 4.800 & $8.953^{\mathrm{c}} \pm 0.409$ & 1.600 & $5.846^{\mathrm{c}} \pm 0.696$ \\
Epinephrine + MMS & 9.467 & $12.277^{\mathrm{b}} \pm 1.345$ & 3.067 & $7.021^{\mathrm{b}} \pm 1.047$ \\
\hline Note: Values are based on the average of readings of 5 slides per mouse per 5 trials \\
Means having the same letter are not significantly different at $\alpha=0.05$ DMRT
\end{tabular}

The possible mechanism of the molecular interaction of the alkylating agent $\left(\mathrm{CH}_{3}{ }^{+}\right)$with DNA is shown Fig. 1. When guanine is alkylated at N-7 (Fig. 2), apurinic sites were produced and this will happen if repair is not carried out immediately.
These apurinic sites may lead to the destabilization of base pairs of the DNA. It has also been found that when alkylation takes place at 0-6 (Fig. 3) of the purine base, guanine, mispairs could induce destabilization of the DNA, leading to a chromosome 
breakage. Alkylation does not only weaken the hydrogen bonds between the guanine and cytosine base pairs but also weakens the $\mathrm{N}-\mathrm{C}$ bonds glycosidic bond.

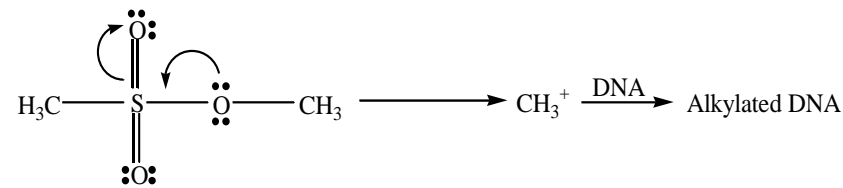

Fig. 1: Metabolic activation of methyl methanesulfonate

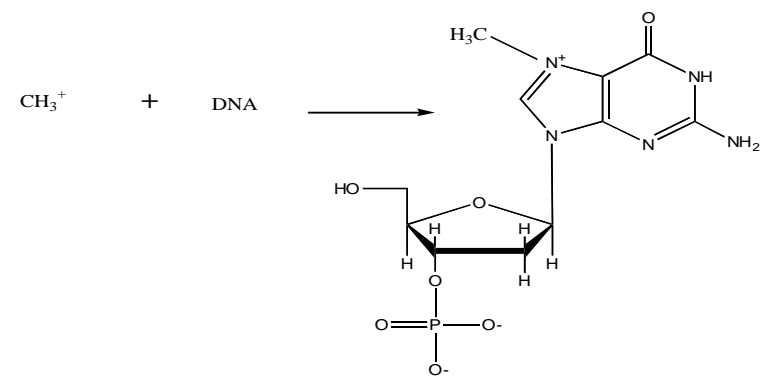

Fig. 2: N-7 alkylation of guanine base

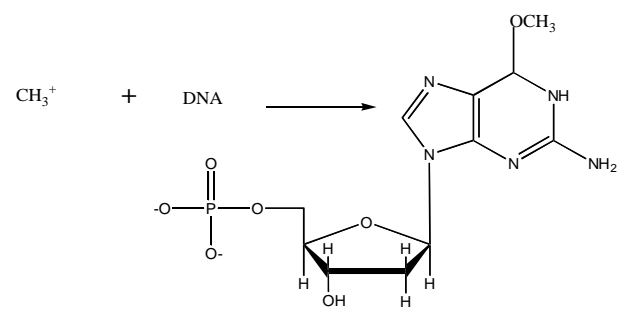

Fig. 3: 0-6 alkylation of guanine base

Figs. 4, 5, 6, and 7 shows the possible mechanism of action of the test substances towards the chromosome breaking effects induced by methyl methanesulfonate.
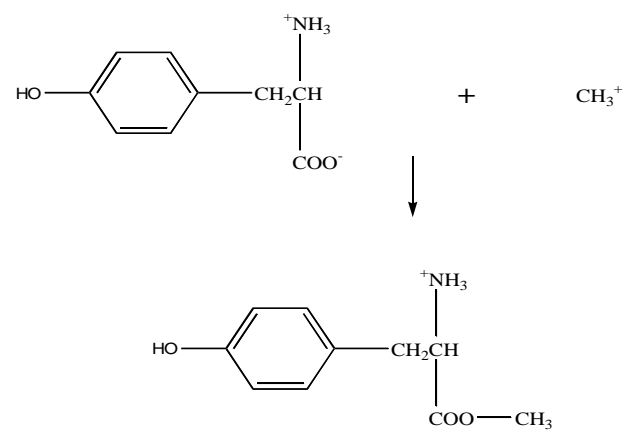

Fig. 4: Possible mechanism of action of $L$-Tyrosine towards MMS<smiles>NC(N)C(=O)O</smiles><smiles>COC(=O)C(N)N</smiles>

Fig. 5: Possible mechanism of action of $L$-DOPA towards MMS

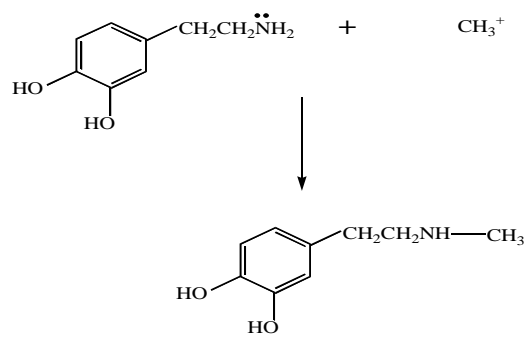

Fig. 6: Possible mechanism of action of dopamine towards MMS<smiles>CCCC(O)c1ccc(O)c(O)c1</smiles>

Fig. 7: Possible mechanism of action of epinephrine towards MMS

Another possible mechanism of action on trapping the carbocation of the alkylating agent by the test substances is through electrophilic substitution on the aromatic ring. The electron cloud of the pi system of the benzene ring of the test substances can attract the carbocation; hence substitution of the hydrogen atom can take place, thus preventing the carbocation $\left(\mathrm{CH}_{3}{ }^{+}\right)$from alkylating the N-7 and 0-6 of the guanine base of the DNA (Fig. 8).
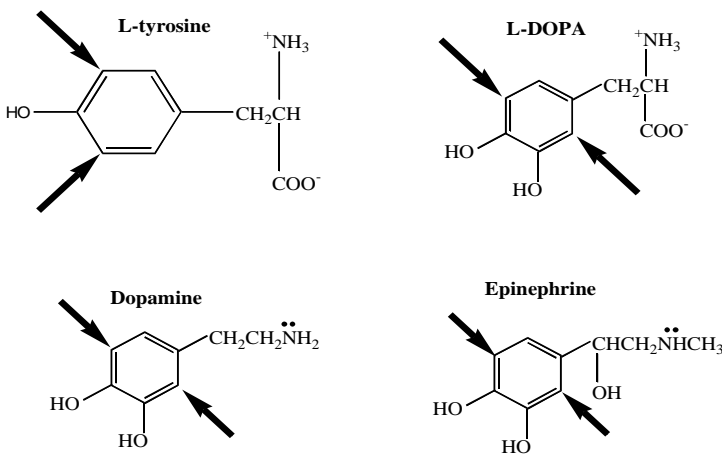

Fig. 8: Possible sites for the interaction of the methyl carbocation and the test compounds

The next following Figs. 9, 10, 11, and 12 will show the electrophilic substitution on the benzene ring of the respective test compounds.
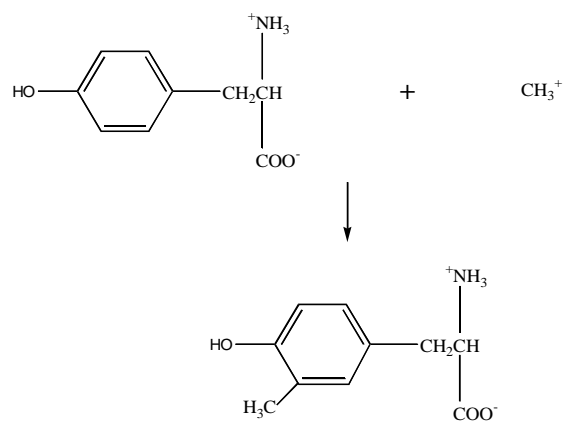

Fig. 9: Electrophilic substitution at the ortho position of $L$ tyrosine 
<smiles>NC(N)C(=O)O</smiles><smiles>[131I-]</smiles><smiles>Cc1ccc(O)c(O)c1C(=O)NC(C)C(=O)O</smiles>

Fig. 10: Electrophilic substitution at the ortho position of $L$-DOPA<smiles>NCCc1ccc(O)c(O)c1</smiles>

$$
+
$$<smiles>Cc1c(O)ccc(CCN)c1O</smiles>

Fig. 11: Electrophilic substitution at the ortho position of dopamine<smiles>CCC(O)c1ccc(O)c(O)c1</smiles><smiles>CNC(O)c1ccc(O)c(O)c1C</smiles>

Fig. 12: Electrophilic substitution at the ortho position of epinephrine

Generally the results show that $L$-Tyrosine has the highest inhibitory effects on the chromosome damaging capacity of methyl methanesulfonate both on the peripheral blood reticulocytes and bone marrow cells. It is also found that epinephrine has the lowest inhibitory effects among the test substances both in peripheral blood reticulocytes and bone marrow cells.

The low inhibitory activity of epinephrine may be accounted to the lack of carboxylate group on its structure and less availability of the lone pair in the amino group of the $\beta$-carbon.

\subsection{In vitro effect}

The effects of $L$-Tyrosine and its metabolites on the mutagenic activity induced by methyl methanesulfonate was assessed on the number of growth of revertants using Ames test utilizing TA98 and TA104 as its test microorganisms.
Overall, Table 3 shows that the test substances are significantly different from the positive control in both TA98 and TA104 strains which implies that the test compounds did not induced reversion of the his to his $^{+}$strains. This suggests that the test compounds are not mutagenic.

Table 3: Reversion of the standard tester strain TA98 and TA104 by $L$-Tyrosine and its metabolites

\begin{tabular}{ccc}
\hline \multirow{2}{*}{ Test componds } & \multicolumn{2}{c}{ Microorganisms } \\
\cline { 2 - 3 } & Transformed data & Transformed data \\
\hline Distilled water & $21.111^{\mathrm{b}} \pm 5.810$ & $13.395^{\mathrm{b}} \pm 3.025$ \\
MMS & $69.933^{\mathrm{a}} \pm 1.010$ & $55.753^{\mathrm{a}} \pm 3.005$ \\
L-Tyrosine & $2.122^{\mathrm{d}} \pm 0.000$ & $5.3999^{\mathrm{bc}} \pm 4.185$ \\
$L$-DOPA & $4.474^{\mathrm{d}} \pm 4.075$ & $9.054^{\mathrm{bc}} \pm 4.813$ \\
Dopamine & $4.704^{\mathrm{d}} \pm 4.474$ & $2.121^{\mathrm{c}} \pm 0.000$ \\
Epinephrine & $12.511^{\mathrm{c}} \pm 5.602$ & $10.009^{\mathrm{bc}} \pm 6.846$ \\
\hline Note: Values are based on the average of readings of 3 replicates \\
per 3 trials. Means having the same letter are not significantly \\
\multicolumn{3}{c}{ different at $\alpha=0.05$ DMRT. }
\end{tabular}

The test compounds did reduce and inhibit the number of revertants induced by methyl methanesulfonate both in the standard tester strains TA98 and TA104 as shown in Table 4. This suggests that the test compounds have antimutagenic potentials. As such, epinephrine accounted the lowest activity in reducing and inhibiting the number of revertants induced by MMS both in the standard tester strains TA98 and TA104.

Generally, $L$-tyrosine and $L$-DOPA in standard tester strain TA98, and $L$-tyrosine, $L$-DOPA, and dopamine in standard tester strain TA104 has the highest activity in reducing the number of revertants induced by MMS.

Table 4: Reversion of the standard tester strain TA98 and TA104 by $L$-tyrosine and its metabolites plus MMS

\begin{tabular}{ccc}
\hline & \multicolumn{2}{c}{ Microorganisms } \\
\cline { 2 - 3 } Test compounds & TA98 revertants & TA104 revertants \\
& Transformed data \pm & Transformed data \pm \\
& S.D. & S.D. \\
\hline Distilled water & $21.111^{\mathrm{b}} \pm 5.810$ & $13.395^{\mathrm{b}} \pm 3.025$ \\
MMS & $69.933^{\mathrm{a}} \pm 1.010$ & $55.753^{\mathrm{a}} \pm 3.005$ \\
$L$-tyrosine+MMS & $24.241^{\mathrm{c}} \pm 5.089$ & $24.216^{\mathrm{c}} \pm 4.621$ \\
L-DOPA+MMS & $27.465^{\mathrm{c}} \pm 0.950$ & $24.347^{\mathrm{c}} \pm 1.816$ \\
Dopamine+MMS & $12.104^{\mathrm{d}} \pm 2.424$ & $25.793^{\mathrm{c}} \pm 1.777$ \\
Epinephrine+MMS & $42.250^{\mathrm{b}} \pm 3.927$ & $37.467^{\mathrm{b}} \pm 0.594$ \\
\hline
\end{tabular}

\section{Conclusion}

Indeed, the use of the amino acid $L$-Tyrosine and its metabolites, $L$-DOPA, dopamine and epinephrine, were proven to be an attractive, effective and promising approach in reducing the number of micronuclei and number of revertants induced by MMS. In general, $L$-Tyrosine and its metabolites have an antimutagenic potential.

\section{Acknowledgment}

The author would like to thank the Department of Science and Technology, Philippines for funding this research. 


\section{References}

Aida M, Birukawa Y, Hachiya N, QUAN T, Suma J, Takizawa Y, Tanaka N, Kuramochi M, Seki H, Tazawa T, and Amano A (1995). Protocol recommended by the CSGMT/JEMS. MMS for the Short-Term mouse peripheral-blood micronucleus test. Mutagenesis, 10(3): 153-159.

Ames BN, Durston WE, Yamasaki E, and Lee FD (1973). Carcinogens are mutagens: a simple test system combining liver homogenates for activation and bacteria for detection. Proceedings of the National Academy of Sciences, 70(8): 2281-2285.

Bajo LM (1994). The effect of metabolism on the antigenotoxic activity of L-Phenylalanine. M.Sc. Thesis, Mindanao State University, Iligan, Philippines.

CSGMT (1990). Single versus multiple dosing in the micronucleus test: the summary of the fourth collaborative study by CSGMT/JEMS.MMS. Collaborative Study Group for the Micronucleus Test (CSGMT), Mutation Research/ Environmental Mutagenesis and Related Subjects, 234(3-4): 205-222.

Fenech M (2000). The in vitro micronucleus technique. Mutation Research/Fundamental and Molecular Mechanisms of Mutagenesis, 455(1): 81-95.

Hayashi M, Sofuni T, and Ishidate M (1983). An application of acridine orange fluorescent staining to the micronucleus test. Mutation Research Letters, 120 (4): 241-247.

Heddle JA (1973). A rapid in vivo test for chromosomal damage. Mutation Research/Fundamental and Molecular Mechanisms of Mutagenesis, 18(2): 187-190.
Magee PN, Montesano R, and Preussmann R (1976). N-Nitroso compounds and related carcinogens. Chemical Carcinogens, 173: 491-625.

Margison GP, Koref MFS, and Povey AC (2002). Mechanism of carcinogenicity/chemotheraphy by $\mathrm{O}^{6-}$ methylguanine. Mutagenesis, 17(6): 483-487.

Maron DM and Ames BN (1983). Revised methods for the Salmonella mutagenicity test. Mutation Research/ Environmental Mutagenesis and Related Subjects, 113(3-4): 173-215.

Mortelmans $\mathrm{K}$ and Zeiger E (2000). The Ames Salmonella/microsome mutagenicity assay. Mutation Research/Fundamental and Molecular Mechanisms of Mutagenesis, 455(1): 29-60.

Schmid W (1976). The micronucleus test for cytogenetic analysis. Chemical Mutagens Principles and Methods for their Detection, 4: 31-53.

Sylianco CYL (1991). Genotoxic and antigenotoxic activities in Philppine medicinal plants. Faruqui AM, Hassan M, and Sandri G (Eds.), Role of women in the development of science and technology in the Third World: 809-811. World Scientific Publication Co., Singapore, Singapore.

Sylianco CYL (1998). Philippine science encyclopedia: Pharmaceutical and chemical sciences. National Research Council of the Philippine, Metro Manila, Philippines.

Sylianco CYL and Guevara AP (1985). Effects of vitamins and mineral ions on genotoxicity of some carcinogens. CORE, 1: 37-56.

Sylianco CYL and Guevara AP (1989). Inhibitory effects of amino acids on somatic and germ cell genotoxicity of some anticancer agents. Trans Transactions of the National Academy of Science and Technology, 11: 233-242. 